\title{
Magnetic resonance guided focused ultrasound surgery (MRgFUS) treatment of osteoid osteoma: a prospective development study
}

\author{
Alessandro Napoli*, Fulvio Zaccagna, Gaia Cartocci, Brachetti Giulia, Gianluca Caliolo, Fabrizio Andrani, \\ Carlo Catalano \\ From Current and Future Applications of Focused Ultrasound 2014. 4th International Symposium \\ Washington, D.C, USA. 12-16 October 2014
}

\section{Background/introduction}

To investigate mid- to long-term efficacy of MRgFUS in the treatment of symptomatic osteoid osteomas.

\section{Methods}

This prospective, IRB approved study involved 29 consecutive patients with clinical and imaging diagnosis of Osteoid Osteoma; all patients underwent MRgFUS ablation (ExAblate, InSightec; Discovery 750 MR unit, GE). Lesions located in vertebral body were excluded; prior RFA or surgery was not considered an exclusion criteria. Patients received therapy using MRgFUS, delivered toward the nidus, identified on MRI and/or CT. Primary endpoints were adverse events (serious and otherwise) and pain relief assessed using questionnaires on Visual Analog Pain Score (VAS) and daily intake of Non-steroidal drugs (NSAIDs). Patient's follow-up, including clinical and imaging examinations, was established at 1,12 and 24 months. As secondary endpoint, imaging examinations (CT and CE-MRI; Gd-BOPTA, Bracco) were used to evaluate inflammatory status after treatment and bone remodeling.

\section{Results and conclusions}

29 patients (female 8; male, 21; mean age 23) were recruited for MRgFUS treatment; all safely completed the procedure. The treatment was well tolerated by all patients and no adverse events were recorded after and during 12-24 months follow-up period. A mean number of $4 \pm 1.8$ sonications with mean energy of $894 \pm 209 \mathrm{~J}$ was necessary to complete the treatment. Three patients underwent staged treatment (1 post-RFA, 1 post surgery, 1 intrarticular position). Complete clinical response was found in $27 / 29$ (93\% CI 6-18) patients in term of pain absence and no intake of NSAIDs. There was a statistically significant difference $(\mathrm{p}=0,001)$ between baseline $(7,9 \pm 1,4)$ and follow-up values $(0,7 \pm 0,1)$ for pain severity, according to VAS. Two patients $(0.6 \%)$ reported pain recurrence requiring both RFA. Imaging evaluation with CE-MRI demonstrated edema and hyperemia decrease in every lesion associated with complete response. At CT, bone remodeling was evident in all complete responders $(27 / 29)$; in $15 / 27$ (55\%) patients, nidus fading was demonstrated. MRgFUS can be safely and effectively adopted for the treatment of Osteoid Osteoma. This application is totally non-invasive, carried out in a single session and with pain relief attainable since the following day after treatment. Our results also indicated a positive trend to bone restoration.

Published: 30 June 2015

\section{doi:10.1186/2050-5736-3-S1-044}

Cite this article as: Napoli et al:: Magnetic resonance guided focused ultrasound surgery (MRgFUS) treatment of osteoid osteoma: a prospective development study. Journal of Therapeutic Ultrasound 2015 3(Suppl 1):O44.

University of Rome - Sapienza, Rome, Italy 